\title{
ON THE TRIEBEL-LIZORKIN SPACE BOUNDEDNESS OF MARCINKIEWICZ INTEGRALS ALONG COMPOUND SURFACES
}

\author{
FENG LIU
}

Abstract. In this paper the author present the boundedness of Marcinkiewicz integral operators associated to compound surfaces with rough kernels given by $h \in \Delta_{\gamma}\left(\mathbb{R}_{+}\right)$and $\Omega \in$ $L\left(\log ^{+} L\right)^{1 / 2}\left(S^{n-1}\right) \cup\left(\cup_{1<r<\infty} B_{r}^{0,-1 / 2}\left(S^{n-1}\right)\right)$ on Triebel-Lizorkin spaces and Besov spaces. The main results of this paper represent improvements as well as natural extensions of many previously known results.

Mathematics subject classification (2010): 42B20, 42B15, 47G10.

Keywords and phrases: Marcinkiewicz integrals, rough kernels, compound surfaces, Triebel-Lizorkin spaces, Besov spaces.

\section{REFERENCES}

[1] H. M. Al-QAssem And A. J. Al-S Alman, A note on Marcinkiewicz integral operators, J. Math. Anal. Appl. 282, 2 (2003), 698-710.

[2] A. Al-Salman, H. Al-Qassem, L. C. Cheng And Y. Pan, $L^{p}$ bounds for the function of Marcinkiewicz, Math. Res. Lett. 9, 5 (2002), 697-700.

[3] H. Al-QASSEM AND Y. PAn, On certain estimates for Marcinkiewicz integrals and extrapolation, Collect. Math. 60, 2 (2009), 123-145.

[4] Y. Ding, D. FAN AND Y. PAN, $L^{p}$-boundedness of Marcinkiewicz integrals with Hardy space function kernel, Acta Math. Sin. (Engl. Ser.), 16, 4 (2000), 593-600.

[5] Y. Ding, D. FAn AND Y. PAN, On the $L^{p}$ boundedness of Marcinkiewicz integrals, Michigan Math. J. 50, (2002), 17-26.

[6] Y. Ding, S. LU AND K. YABUtA, A problem on rough parametric Marcinkiewicz functions, J. Austr. Math. Soc. 72, 1 (2002), 13-21.

[7] Y. Ding And Y. Pan, $L^{p}$ bounds for Marcinkiewicz integrals, Proc. Edinb. Math. Soc. 46, 3 (2003), 669-677.

[8] Y. Ding, Q. XUE AND K. YABUtA, Boundedness of the Marcinkiewicz integrals with rough kernel associated to surfaces, Tohoku Math. J. 62, 2 (2010), 233-262.

[9] M. FrAZIER, B. JAWERTH AND G. WEISS, Littlewood-Paley theory and the study of function spaces, CBMS Reg. Conf. Ser. vol. 79, Amer. Math. Soc., Providence, RI 1991.

[10] D. FAN AND Y. PAN, Singular integral operators with rough kernels supported by subvarieties, Amer. J. Math. 119, 4 (1997), 799-839.

[11] HÖRMANDER, Estimates for translation invariant operators in $L^{p}$ spaces, Acta Math., 104, 10 (1960), 93-104.

[12] F. LiU, Integral operators of Marcinkiewicz type on Triebel-Lizorkin spaces, Math. Nachr., (to appear) (2016). DOI: 10.1002/mana.201500374.

[13] F. LiU AND H. WU, Multiple singular integrals and Marcinkiewicz integrals with mixed homogeneity along surfaces, J. Inequ. Appl. 2012, 189 (2012), 1-23.

[14] F. LiU AND H. WU, On Marcinkiewicz integrals associated to compound mappings with rough kernels, Acta Math. Sin. (Engl. Ser.), 30, 7 (2014), 1210-1230.

[15] F. LiU And D. Zhang, Parabolic Marcinkiewicz integrals associated to polynomials compound curves and extrapolation, Bull. Korean Math. Soc. 52, 3 (2015), 771-788. 
[16] R. RICCI AND E. M. STEIN, Harmonic analysis on nilpotent groups and singular integrals I: Oscillatory integrals, J. Funct. Anal. 73, 1 (1987), 179-184.

[17] M. Sakamoto AND K. Yabuta, Boundedness of Marcinkiewicz functions, Studia Math. 135, (1999), 103-142.

[18] E. M. Stein, On the function of Littlewood-Paley, Lusin and Marcinkiewicz, Trans. Amer. Math. Soc. 88, 2 (1958), 430-466.

[19] H. TRIEBEL, Theory of Function Spaces, Monogr. Math. vol. 78, Birkhäser Verlag, Basel, 1983.

[20] K. YABUTA, Triebel-Lizorkin space bounddedness of Marcinkiewicz integrals associated to surfaces, Appl. Math. J. Chinese Univ. 30, 4 (2015), 418-446.

[21] C. Zhang AND J. Chen, Boundedness of $g$-functions on Triebel-Lizorkin spaces, Taiwanese J. Math. 13, 3 (2009), 973-981.

[22] C. Zhang And J. Chen, Boundedness of Marcinkiewicz integral on Triebel-Lizorkin spaces, Appl. Math. J. Chinese Univ. Ser. B, 25, 25 (2010), 48-54. 